\title{
Theoretical study of temperature elevation at muscle/bone interface during ultrasound hyperthermia
}

\author{
Win-Li Lin \\ Institute of Biomedical Engineering, National Taiwan University, Taipei, Taiwan \\ Chinng-Tsung Liauh \\ Department of Mechanical Engineering, Kung-shan Institute of Technology, Tainan, Taiwan \\ Yung-Yaw Chen \\ Department of Electrical Engineering, National Taiwan University, Taipei, Taiwan \\ Hwa-Chang Liu and Ming-Jium Shieh ${ }^{\text {a) }}$ \\ Institute of Biomedical Engineering, National Taiwan University, Taipei, Taiwan
}

(Received 15 July 1999; accepted for publication 9 February 2000)

This paper examines the distributions of the SAR (specific absorption rate) ratio and temperature elevation when an ultrasound beam propagates through the interface of muscle and bone. This interface is regarded as a flat boundary to partition the energy of the ultrasound beam, and the analytical solution of temperature distribution is based on the steady-state bio-heat transfer equation. The parameters considered are the incident angle of ultrasound beam, the ultrasound frequency, the acoustic attenuation coefficients of refracted longitudinal and shear waves in bone, and the blood perfusion in muscle. The results show that the peak of the SAR ratio is always at the interface of muscle and bone, while the peak of temperature is located in the bone region beyond the interface. A muscle with lower perfusion or a bone with higher acoustic attenuation results in the shifting of the temperature peak closer to the interface. It is more difficult to heat a higher perfused muscle in front of a bone using a lower frequency ultrasound since the temperature elevation for bone relative to muscle is greater. (C) 2000 American Association of Physicists in Medicine. [S0094-2405(00)01005-1]

Key words: ultrasound hyperthermia, muscle/bone interface, absorbed power deposition, temperature elevation

\section{INTRODUCTION}

The steady-state temperature rise for a focused ultrasonic beam along its axis was performed at the soft tissue/bone interface with normal incidence ${ }^{1}$ and the temperature for different sets of transducer's diameter, frequency and curvature's radius ${ }^{2}$ has been studied. The results showed that the maximum temperature rise along the beam axis occurs at a point in the bone side, close to the interface, or in the focal zone. Further study with a focused transducer for an agar/ bone phantom has been performed by Fujii et $\mathrm{al}^{3}{ }^{3}$ to investigate the temperature rise at the muscle/bone interface. Their results showed that the peak temperatures were mainly at the interface even though the focal point was not located at the interface and the incident angle was an important factor when the focal point was located at the interface.

Several studies ${ }^{4-7}$ have shown the preferential heating of the post target bone with nonfocused physiotherapy ultrasound transducers. The in vivo experimental results for Lehmann et al. ${ }^{6}$ showed that the peak temperature occurred in the spongy bone when the hind legs of pigs were exposed for $5 \mathrm{~min}$ to continuous unfocused ultrasound of a $1.0 \mathrm{MHz}$ frequency. Chan et al. ${ }^{8,9}$ theoretically studied the heat generation in a fat-muscle-bone model by planar ultrasound wave. They determined that the incident angle of the ultrasound beam should be larger than $\sim 60^{\circ}$ in order to avoid both absorption and shear wave generation, and the effect of shear wave generation was especially significant for incident angles between $30^{\circ}$ and $50^{\circ}$. Besides, Moros et al..$^{10}$ developed an ultrasound power deposition model for the chest wall based on secondary-source and plane-wave theories. They showed that the determining factors for the power deposition are reflection at major interfaces and attenuation in bone, and the incident angle of the ultrasound beam determined the dominance of the factors.

Similar hot spots also appear in the posttarget bone during ultrasound hyperthermia when weakly focused, low frequency, single or multiple beam systems are used to heat tissues in front of bones. ${ }^{11-15}$ Hynynen and DeYoung ${ }^{14}$ studied the influence of a muscle/bone interface on the temperature distribution in vivo dogs' thighs during scanned, focused ultrasound hyperthermia. The steady-state temperature distributions showed that the peak value was located at the interface of muscle/bone when they used $10-\mathrm{mm}$ spacing multisensors thermal probes to measure the temperatures and cubic-spline interpolation to obtain the isothermal lines in the plane.

The absorbed power deposition and the bio-heat transfer determine the temperature elevation around the interface of muscle and bone. The shape and incident angle of the ultrasound beam and the acoustic properties of muscle/bone 
configure the pattern of absorbed power deposition. During external ultrasound hyperthermia for treating a tumor located in front of a bone, it is necessary to prevent overheating not only the intervening normal tissue between the skin and the target volume but also the bone behind the tumor. Thus, a convolution power deposition with a convergent-divergent cone shape should be formed to provide appropriate heating. ${ }^{16}$ Scanned, focused ultrasound heating systems ${ }^{17-21}$ can produce a convolution power deposition close to this ideal shape by scanning the ultrasound beams with appropriate scan parameters. In addition, two-dimensional arrays of transducers ${ }^{22-24}$ can yield such a power deposition by controlling the amplitude and phase of each array element. The power deposition for ultrasound hyperthermia is different from those mentioned earlier, especially for a single focused ultrasound transducer. The present work employs a onedimensional model of ultrasound power deposition to simulate and examine the absorbed power deposition in the region across the muscle/bone interface. An analytical solution for the resulting temperature is also studied based on the steadystate bio-heat transfer equation. The incident angle of the ultrasound beam, the ultrasound frequency, and the acoustic attenuation coefficients of refracted longitudinal and shear waves of bone are studied for the SAR ratio distribution (where $\mathrm{SAR}=2 \alpha I, \alpha$ and $I$ are ultrasound absorption coefficient and intensity, respectively). Besides, blood perfusion of muscle is also considered for the further study of the distribution of temperature elevation.

\section{METHOD AND MATERIALS}

\section{A. Power deposition and energy partition at the interface of muscle and bone}

Figure 1(a) shows the geometry of the power deposition during ultrasound hyperthermia. The ultrasound beam propagates from the left side to the right side and the ultrasound power is assumed to propagate within a conical shape for the convolution power deposition. The total ultrasound energy is attenuated according to the exponential law ${ }^{18,25}$ and the deposition of ultrasonic intensity within the cone region is assumed as a uniform distribution at each depth. The portion across the muscle/bone interface is taken for this study and the geometrical variation has been neglected. The deposition of ultrasound energy is regarded as a one-dimensional distribution as shown in Fig. 1(b). The left side from 0 to $z_{b}$, the middle from $z_{b}$ to $z_{c}$, and the right side from $z_{c}$ to $z_{e}$, are regarded as the muscle region, the cortical layer, and the spongy/marrow region of bone, respectively. For the following study, $z_{b}, z_{c}$, and $z_{e}$, are set at $5,5.35$, and $10 \mathrm{~cm}$, respectively. The thickness of the cortical layer is assumed as $0.35 \mathrm{~cm}$. The media of muscle and bone were modeled as liquid-solid and the muscle can be considered as liquid due to the contribution of the shear waves to the heating in the muscle is negligible. ${ }^{26}$ The interface of muscle and bone is regarded as a flat boundary and, according to Snell's law and Mayer, ${ }^{27}$ the energy partition at the interface can be written as follows:

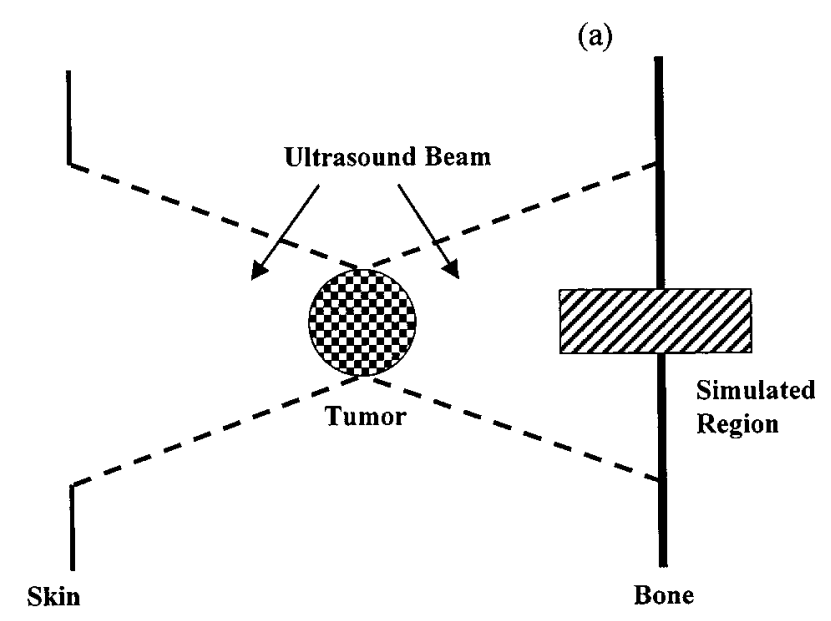

Acoustic Window

(b)

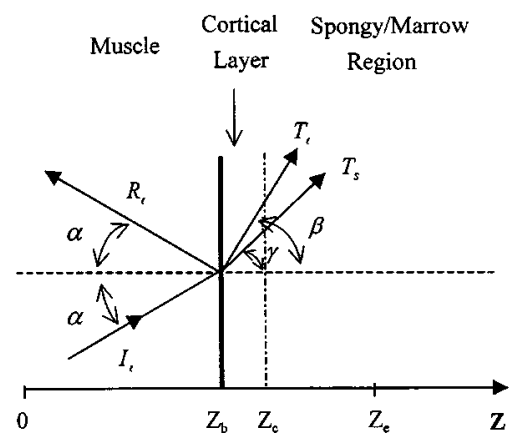

FIG. 1. Schematic diagram of the geometry studied for the temperature elevation distributions of the SAR ratio and temperature around the muscle/ bone interface when the ultrasound beam contacts the bone surface. (a) The power deposition during ultrasound hyperthermia, with the power convergent to the tumor and then divergent. (b) The portion across the interface of muscle/bone as shown in (a) has been taken for the study of the SAR ratio and temperature elevation. The ultrasound beam penetrates the tissues from the left, propagating through the muscle and then impinging against the muscle/bone interface at a distance of $z_{b}$. The cortical layer is from $z_{b}$ to $z_{c}$ and the spongy/marrow region from $z_{c}$ to $z_{e} . \alpha, \beta$, and $\gamma$ are the angles of the incident longitudinal wave $\left(I_{l}\right)$, refracted longitudinal wave $\left(T_{l}\right)$, and refracted shear wave $\left(T_{s}\right)$, respectively. $R,(1-R)(1-S)$, and $(1-R) S$ are the energy partition ratios of $R_{l}$ (reflected longitudinal wave), $T_{l}$, and $T_{s}$, to $I_{l}$, respectively.

$$
\begin{aligned}
& \frac{v_{m}}{\sin \alpha}=\frac{v_{b l}}{\sin \beta}=\frac{v_{b s}}{\sin \gamma}, \\
& R=\left[\frac{1-C \cdot(1-2 A)}{1+C \cdot(1-2 A)}\right]^{2} \\
& (1-R)(1-S)=\frac{4 B \cdot C}{[1+C \cdot(1-2 A)]^{2}}, \\
& (1-R) S=\frac{4 D \cdot\left(\rho_{b} / \rho_{m}\right)}{[1+C \cdot(1-2 A)]^{2}},
\end{aligned}
$$

with

$A=\sin \gamma \cdot \sin 2 \gamma \cdot\left[\cos \gamma-\left(\frac{v_{b s}}{v_{b l}}\right) \cdot \cos \beta\right] ; \quad B=(\cos 2 \gamma)^{2}$ 
TABLE I. Nominal values of acoustic and tissue parameters used in this study [thermal and tissue properties (Refs. 33-36) and acoustic attenuation (Ref. 38)]

\begin{tabular}{lccccc}
\hline \hline & $\begin{array}{c}\text { Thermal } \\
\text { conductivity } \\
(k)\left(\mathrm{W} \mathrm{m}^{-1}{ }^{\circ} \mathrm{C}^{-1}\right)\end{array}$ & $\begin{array}{c}\text { Density } \\
\left(\rho_{m}, \rho_{b}\right) \\
\left(\mathrm{kg} \mathrm{m}^{-3}\right)\end{array}$ & $\begin{array}{c}\text { Blood } \\
\text { perfusion }(W) \\
\left(\mathrm{kg} \mathrm{m}^{-3} \mathrm{~s}^{-1}\right)\end{array}$ & $\begin{array}{c}\text { Velocity } \\
\left(v_{m}, v_{b l}, v_{b s}\right) \\
\left(\mathrm{m} \mathrm{s}^{-1}\right)\end{array}$ & $\begin{array}{c}\text { Attenuation coefficient } \\
\left(\mu_{m}, \mu_{b}, \mu_{b s}\right) \\
\left(\mathrm{Np} \mathrm{m}^{-1} \mathrm{MHz}^{-1}\right)\end{array}$ \\
\hline Muscle & 0.55 & 1050 & 2 & $v_{m}=1500$ & $\mu_{m}=5$ \\
Bone & & & & $v_{b l}=3600$ & $\mu_{b}=86.3$ \\
Cortical & 2.3 & 1700 & 0 & $v_{b s}=1800$ & $\mu_{b s}=126.6$ \\
Spongy/marrow & 0.58 & 1300 & 0.1 & & \\
\hline \hline
\end{tabular}

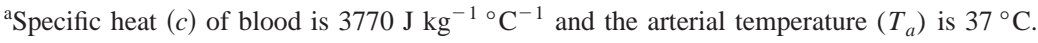

$C=\frac{\rho_{b} v_{b l} \cos \alpha}{\rho_{m} v_{m} \cos \beta} ; \quad D=\left(\frac{v_{b s}}{v_{m}}\right)^{2} \cdot \sin 2 \alpha \cdot \sin 2 \gamma$,

where $v_{m}, v_{b l}$, and $v_{b s}$ are the velocities of the incident longitudinal wave, refracted longitudinal wave, and refracted shear wave, respectively; $\alpha, \beta$, and $\gamma$ are the angles of the incident longitudinal wave, refracted longitudinal wave, and refracted shear wave, respectively; $R,(1-R)(1-S)$, and $(1-R) S$ are the energy ratios of the reflected longitudinal wave, refracted longitudinal wave, and refracted shear wave to the incident longitudinal wave, respectively; $S$ and $(1-S)$ are the energy ratios of the refracted shear and longitudinal waves to the total transmitted wave into the bone, respectively; and $\rho_{m}$ and $\rho_{b}$ are the densities of muscle and bone, respectively.

The ultrasound power deposition is as follows.

(a) For $0 \leqslant z<z_{b}$ (the muscle region),

$$
\begin{aligned}
I(z) & =I(0) \cdot e^{-2 \mu_{m} z}+R \cdot I(0) \cdot e^{-2 \mu_{m}\left(2 z_{b}-z\right)} \\
& \Rightarrow q(z)=2 \mu_{m} I(0) \cdot\left[e^{-2 \mu_{m} z}+R \cdot e^{-2 \mu_{m}\left(2 z_{b}-z\right)}\right],
\end{aligned}
$$

where $I(0)$ and $I(z)$ are the ultrasound intensities at the depth of 0 and $z$, respectively; $q(z)$, heat source function, is the absorbed ultrasonic power density at $z ; \mu_{m}$ is the ultrasound attenuation of muscle; and $z_{b}$ is the depth of the interface of muscle/bone.

(b) For $z_{b} \leqslant z \leqslant z_{e}$ (the bone region),

$$
\begin{aligned}
I(z)= & (1-R) I(0) \cdot e^{-2 \mu_{m} z_{b}} \cdot S \cdot e^{-2 \mu_{b s}\left(z-z_{b}\right)} \\
& +(1-R) I(0) \cdot e^{-2 \mu_{m} z_{b}} \cdot(1-S) \cdot e^{-2 \mu_{b}\left(z-z_{b}\right)} \\
\Rightarrow & q(z)=I(0) \cdot e^{-2 \mu_{m} z_{b}} \cdot\left[2 S(1-R) \mu_{b s} \cdot e^{-2 \mu_{b s}\left(z-z_{b}\right)}\right. \\
& \left.+2(1-S)(1-R) \mu_{b} \cdot e^{-2 \mu_{b}\left(z-z_{b}\right)}\right]
\end{aligned}
$$

where $\mu_{b s}$ and $\mu_{b}$ are the ultrasound attenuations of the refracted shear and longitudinal waves in the bone, respectively.

Assuming that the ultrasound intensities are not strong enough to cause wave distortion and ultrasound propagates in the linear range (nonlinear distortion is neglected), and that the attenuation and absorption coefficients for tissues are equal (all of the attenuated energy is absorbed in the heating field), ${ }^{2,28}$ then we can obtain the ratio of the SAR at any depth of the tissues to that at $z=0$ as

$$
\operatorname{SR}(z ; 0)=\frac{q(z)}{q(0)},
$$

where $\operatorname{SR}(z ; 0)$ is the ratio of the $\mathrm{SAR}$ (specific absorption rate) at the depth of $z$ to that at $z=0 ; q(z)$ for muscle and bone can be obtained from Eqs. (3a) and (3b), and $q(0)$ $=2 \mu_{m} I(0) \cdot\left[1+R \cdot e^{-4 \mu_{m} z_{b}}\right]$ can be obtained from Eq. (3a) by setting $z=0$.

\section{B. Temperature solver}

Pennes ${ }^{29}$ steady-state bio-heat transfer equation (BHTE) was used to solve for the temperature distribution,

$$
k \frac{d^{2} T}{d z^{2}}-W c\left(T-T_{a}\right)+q=0 .
$$

The above BHTE is a simplification that neglects the effects of discrete blood vessels and the redistribution of thermal energy within the local vascular network. However, the BHTE is a good approximation that offers a practical approach for modeling biothermal processes ${ }^{30-32}$ and performing general parameter studies. We selected thermal properties to approximate averages for muscle ${ }^{33-35}$ and bone tissues, ${ }^{36}$ and the values are shown in Table I. The absorbed ultrasonic power density $(q)$ was obtained using the earlier procedure for a given input power. All attenuated ultrasonic energy was assumed to be absorbed by the tissue. The anatomic and physiological properties were assumed to remain constant throughout the entire field, and metabolism was neglected due to its small contribution to the temperature changes. $^{31,37}$ The blood perfusion $(W)$ is maintained at 2, 0 , and $0.1 \mathrm{~kg} \mathrm{~m}^{-1} \mathrm{~s}^{-1}$ for the muscle, cortical layer, and spongy/marrow bone, ${ }^{36}$ respectively, unless otherwise noted.

The bio-heat transfer for each region can be written as

$$
\begin{aligned}
& k_{i} \frac{d^{2} T_{i}}{d z^{2}}-W_{i} c\left(T_{i}-T_{a}\right)+q_{i} \\
& \quad=0 \quad\left\{\begin{array}{lll}
i=1, & 0 \leqslant z<z_{b} & \text { (muscle region) } \\
i=2, & z_{b} \leqslant z<z_{c} & \text { (cortical layer) }, \\
i=3, & z_{c} \leqslant z \leqslant z_{e} & \text { (spongy/marrow bone), }
\end{array}\right.
\end{aligned}
$$


where $k_{i}$ and $W_{\mathrm{i}}$ represent the thermal conductivity and blood perfusion for muscle $(i=1)$, the cortical layer of bone $(i=2)$, and the spongy/marrow bone $(i=3)$; and $q_{i}$ is the absorbed ultrasonic power density which can be obtained by Eq. (3).

The temperature distribution in each region can be obtained by solving Eq. (6) and can be written as

$$
T_{i}=T_{a}+A_{i} e^{\sqrt{F_{i} z}}+B_{i} e^{-\sqrt{F_{i} z}}+\zeta_{i}(z)
$$

with

$$
F_{i}=\frac{W_{i} c}{k_{i}} \text { for } i=1,2,3 \text {; }
$$

$$
\zeta_{i}(z)=\left\{\begin{array}{l}
\frac{2 \mu_{m} I(0)}{k_{i}\left(F_{i}-4 \mu_{m}^{2}\right)} \cdot\left[e^{-2 \mu_{m} z}+R \cdot e^{-2 \mu_{m}\left(2 z_{b}-z\right)}\right] \quad \text { for } i=1, \\
\frac{(1-R) I(0) \cdot e^{-2 \mu_{m} z_{b}}}{k_{i}} \cdot\left[\frac{2 \mu_{b s} S}{\left(F_{i}-4 \mu_{b s}^{2}\right)} \cdot e^{-2 \mu_{b s}\left(z-z_{b}\right)}+\frac{2 \mu_{b}(1-S)}{\left(F_{i}-4 \mu_{b}^{2}\right)} \cdot e^{-2 \mu_{b}\left(z-z_{b}\right)}\right] \text { for } i=2,3,
\end{array}\right.
$$

and $A_{i}$ and $B_{i}$ are the integration constants.

The six boundary conditions are listed as follows,

$$
\begin{aligned}
& T_{1}(0)=T_{a}, \\
& T_{3}\left(z_{e}\right)=T_{a}, \\
& T_{1}\left(z_{b}\right)=T_{2}\left(z_{b}\right), \\
& \left.k_{1} \frac{d T_{1}}{d z}\right|_{z=z_{b}}=\left.k_{2} \frac{d T_{2}}{d z}\right|_{z=z_{b}}, \\
& T_{2}\left(z_{c}\right)=T_{3}\left(z_{c}\right), \\
& \left.k_{2} \frac{d T_{2}}{d z}\right|_{z=z_{c}}=\left.k_{3} \frac{d T_{3}}{d z}\right|_{z=z_{c}} .
\end{aligned}
$$

The above boundary conditions (8a) $-(8 \mathrm{f})$ can be used to solve for the six integration constants $\left(A_{i}, B_{i}\right.$, for $\left.i=1,2,3\right)$ in Eq. (7).

From the boundary conditions (8a), (8b), (8e), $B_{1}, B_{2}$, and $B_{3}$ can be replaced by

$$
\begin{aligned}
B_{1}= & -\left[A_{1}+\zeta_{1}(0)\right], \\
B_{2}= & \left\{A_{3} e^{\sqrt{F_{3}} z_{c}}-\left[A_{3} e^{\sqrt{F_{3}} z_{e}}+\zeta_{3}\left(z_{e}\right)\right] \cdot e^{\sqrt{F_{3}}\left(z_{e}-z_{c}\right)}\right. \\
& \left.-A_{2} e^{\sqrt{F_{2}} z_{c}}+c_{0}\right\} \cdot e^{\sqrt{F_{2}} z_{c}}
\end{aligned}
$$

with $c_{0}=\zeta_{3}\left(z_{c}\right)-\zeta_{2}\left(z_{c}\right)$,

$$
B_{3}=-\left[A_{3} e^{\sqrt{F_{3}} z_{e}}+\zeta_{3}\left(z_{e}\right)\right] \cdot e^{\sqrt{F_{3}} z_{e}} .
$$

Using the above $B_{1}, B_{2}, B_{3}$ and the boundary conditions (8c), (8d), and (8f), we can obtain the following three equations for $A_{1}, A_{2}$, and $A_{3}$ :

$$
\begin{aligned}
& \alpha_{1} A_{1}+\beta_{1} A_{2}+\gamma_{1} A_{3}+\delta_{1}=0, \\
& \alpha_{2} A_{1}+\beta_{2} A_{2}+\gamma_{2} A_{3}+\delta_{2}=0, \\
& \alpha_{3} A_{1}+\beta_{3} A_{2}+\gamma_{3} A_{3}+\delta_{3}=0,
\end{aligned}
$$

with

$$
\alpha_{1}=e^{\sqrt{F_{1}} z_{b}}-e^{-\sqrt{F_{1}} z_{b}}, \quad \beta_{1}=e^{-\sqrt{F_{2}}\left(z_{b}+2 z_{c}\right)}-e^{\sqrt{F_{2}} z_{b}},
$$

$$
\gamma_{1}=-a_{0} e^{-\sqrt{F_{2}} z_{b}}, \quad \delta_{1}=d_{0}+b_{0} e^{-\sqrt{F_{2}} z_{b}},
$$$$
\alpha_{2}=k_{1} \sqrt{F_{1}} \cdot\left(e^{\sqrt{F_{1}} z_{b}}+e^{-\sqrt{F_{1}} z_{b}}\right),
$$$$
\beta_{2}=-k_{2} \sqrt{F_{2}} \cdot\left[e^{-\sqrt{F_{2}}\left(z_{b}+2 z_{c}\right)}+e^{\sqrt{F_{2}} z_{b}}\right],
$$
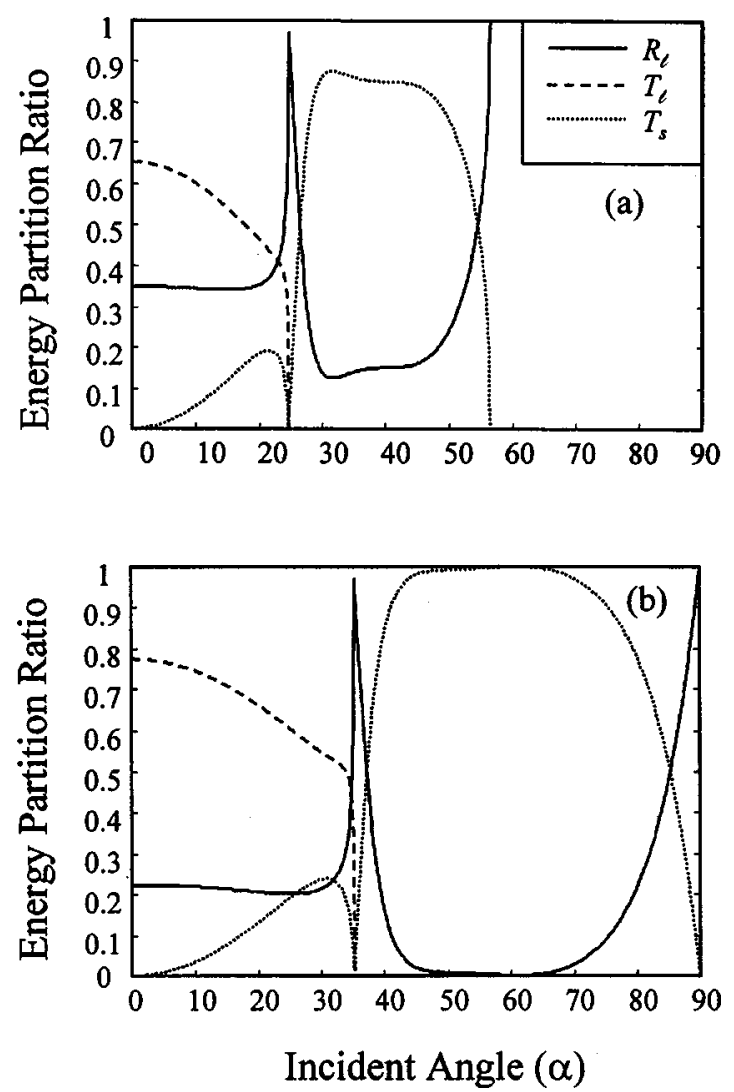

FIG. 2. Energy partition ratios plotted against the incident angle of ultrasound beam at the interface of muscle/bone. The densities of muscle and cortical bone are 1050 and $1700 \mathrm{~kg} \mathrm{~m}^{-3}$, respectively. (a) The velocities of the incident longitudinal wave $\left(v_{m}\right)$, and the refracted longitudinal $\left(v_{b l}\right)$ and shear $\left(v_{b s}\right)$ waves are 1500,3600 , and $1800 \mathrm{~m} \mathrm{~s}^{-1}$, respectively; (b) $v_{b l}$ is equal to $2600 \mathrm{~m} \mathrm{~s}^{-1}$ with $v_{b s}$ half of $v_{b l}$. 
TABLE II. Cases of incident angle and energy partition for the study of the SAR ratio and temperature distribution: $\alpha$ is the angle of incident longitudinal wave at the interface of muscle/bone; $R$ is the energy ratio of the reflected longitudinal wave to the incident longitudinal wave; and $S$ is the energy ratio of the refracted shear wave to the total transmitted wave into the bone.

\begin{tabular}{ccll}
\hline \hline Case & $\alpha$ & $R$ & $S$ \\
\hline 1 & Ideal & 0.0 & 0.0 \\
2 & $0^{\circ}$ & 0.35 & 0.0 \\
3 & $20^{\circ}$ & 0.35 & 0.27 \\
4 & $40^{\circ}$ & 0.2 & 1.0 \\
5 & $60^{\circ}$ & 1.0 & 0.0 \\
\hline
\end{tabular}

$$
\begin{aligned}
\gamma_{2}= & a_{0} k_{2} \sqrt{F_{2}} \cdot e^{-\sqrt{F_{2}} z_{b}}, \quad \delta_{2}=k_{1} \sqrt{F_{1}} \zeta_{1}(0) \cdot e^{-\sqrt{F_{1}} z_{b}} \\
& +k_{1} \zeta_{1}^{\prime}\left(z_{b}\right)-k_{2} \zeta_{2}^{\prime}\left(z_{b}\right)-b_{0} k_{2} \sqrt{F_{2}} \cdot e^{-\sqrt{F_{2}} z_{b}}, \\
\alpha_{3}= & 0, \quad \beta_{3}=2 k_{2} \sqrt{F_{2}} \cdot e^{\sqrt{F_{2}} z_{c}}, \\
\gamma_{3}= & -\left[k_{2} a_{0} \sqrt{F_{2}} \cdot e^{-\sqrt{F_{2}} z_{c}}+k_{3} \sqrt{F_{3}} \cdot e^{\sqrt{F_{3}} z_{c}}\right. \\
& \left.+k_{3} \sqrt{F_{3}} \cdot e^{\sqrt{F_{3}}\left(2 z_{e}-z_{c}\right)}\right], \\
\delta_{3}= & b_{0} k_{2} \sqrt{F_{2}} \cdot e^{-\sqrt{F_{2}} z_{c}}+k_{2} \zeta_{2}^{\prime}\left(z_{c}\right) \\
& -k_{3} \zeta_{3}^{\prime}\left(z_{c}\right)-k_{3} \sqrt{F_{3}} \zeta_{3}\left(z_{e}\right) \cdot e^{\sqrt{F_{3}}\left(z_{e}-z_{c}\right)},
\end{aligned}
$$
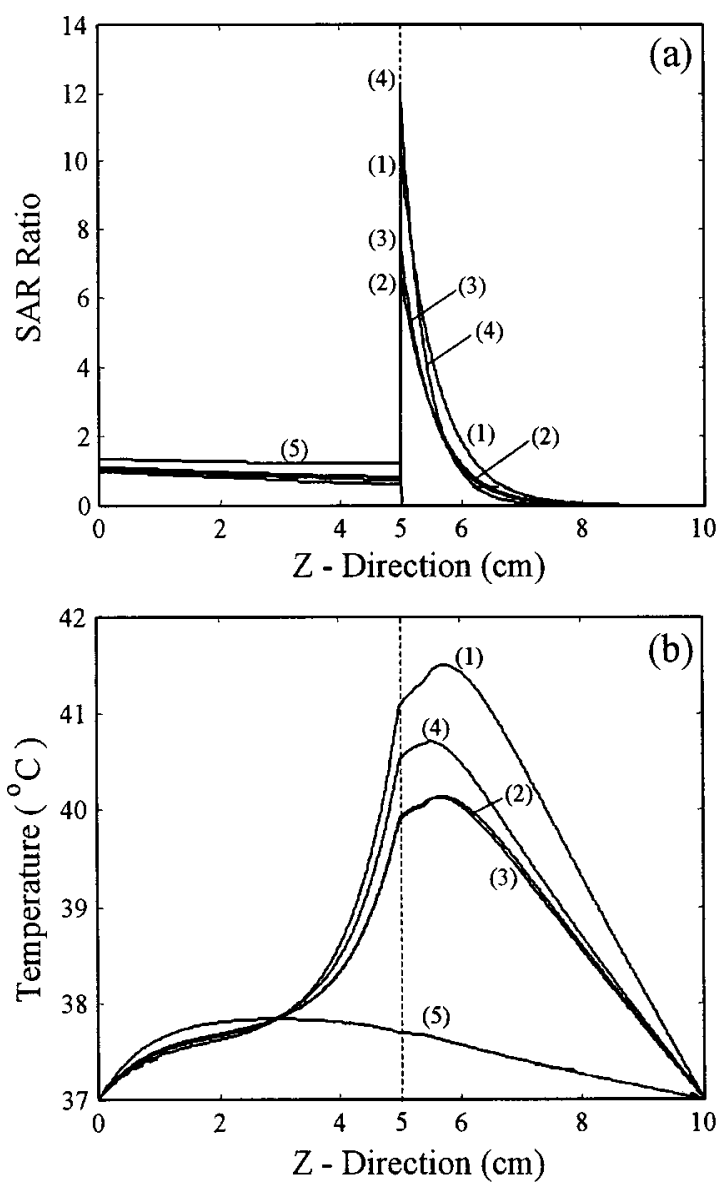

and

$$
\begin{aligned}
& a_{0}=e^{\left(\sqrt{F_{2}}+\sqrt{F_{3}}\right) z_{c}}-e^{\sqrt{F_{2}} z_{c}+2 \sqrt{F_{3}} z_{e}}, \\
& b_{0}=\zeta_{3}\left(z_{e}\right) \cdot e^{\sqrt{F_{2}} z_{c}+\sqrt{F_{3}}\left(z_{e}-z_{c}\right)}-c_{0} e^{\sqrt{F_{2}} z_{c}}, \\
& c_{0}=\zeta_{3}\left(z_{c}\right)-\zeta_{2}\left(z_{c}\right), \\
& d_{0}=\zeta_{1}\left(z_{b}\right)-\zeta_{2}\left(z_{b}\right)-\zeta_{1}(0) \cdot e^{-\sqrt{F_{1}} z_{b}} .
\end{aligned}
$$

The analytical solution of the temperature distribution in each region can be obtained by solving for $A_{1}, A_{2}, A_{3}, B_{1}$, $B_{2}$, and $B_{3}$.

\section{RESULTS}

The relationship between the incident wave and the reflected/refracted waves at the interface of muscle and bone is determined by Eqs. (1) and (2), ${ }^{27}$ which are related to the velocities of waves in tissues and the tissue densities. Figure 2(a) shows the energy partition ratios of the reflected wave and the refracted longitudinal and shear waves to the incident wave when an ultrasound beam propagates at the interface of muscle and bone with different incident angles. The acoustic and tissue parameters used in this study are shown in Table I. Figure 2(a) reveals that the energy ratio of the refracted longitudinal wave decreases but increases for the shear wave as the incident angle of the ultrasound beam at the interface
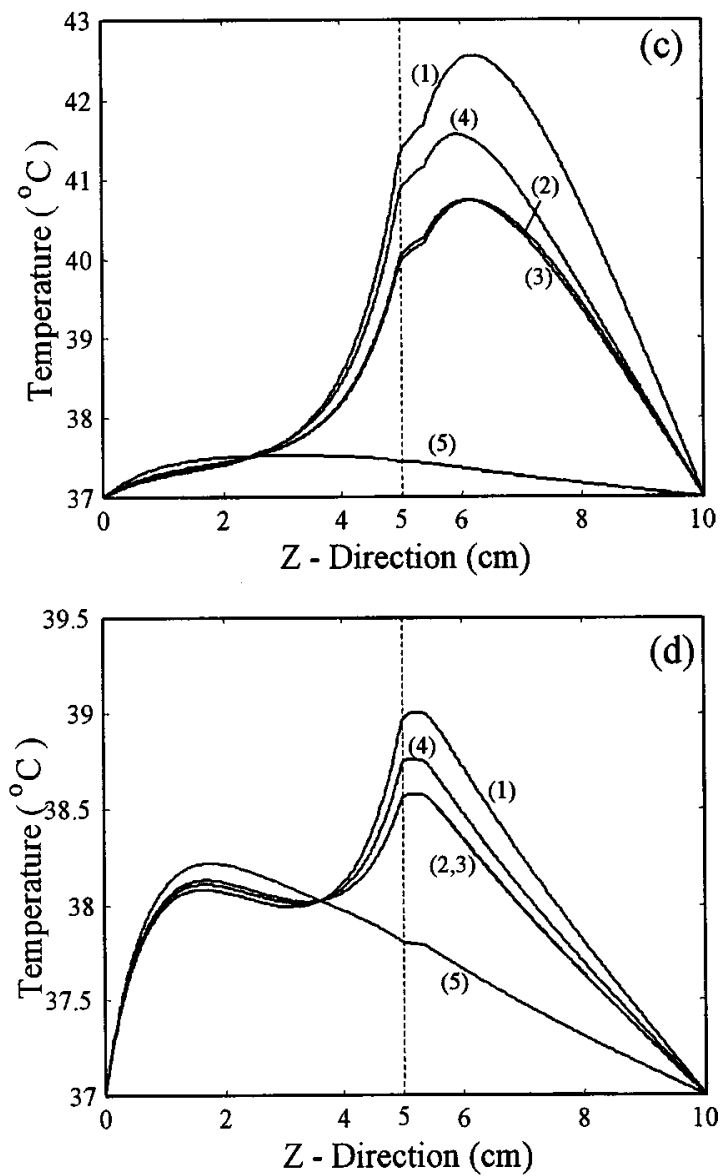

FIG. 3. (a) and (b) Distributions of the SAR ratio and temperature in the tissues for the cases in Table II, with ultrasound frequency 1.0 MHz. (c) and (d) Temperature distributions for frequency equal to 0.5 or $3.0 \mathrm{MHz}$, respectively. 

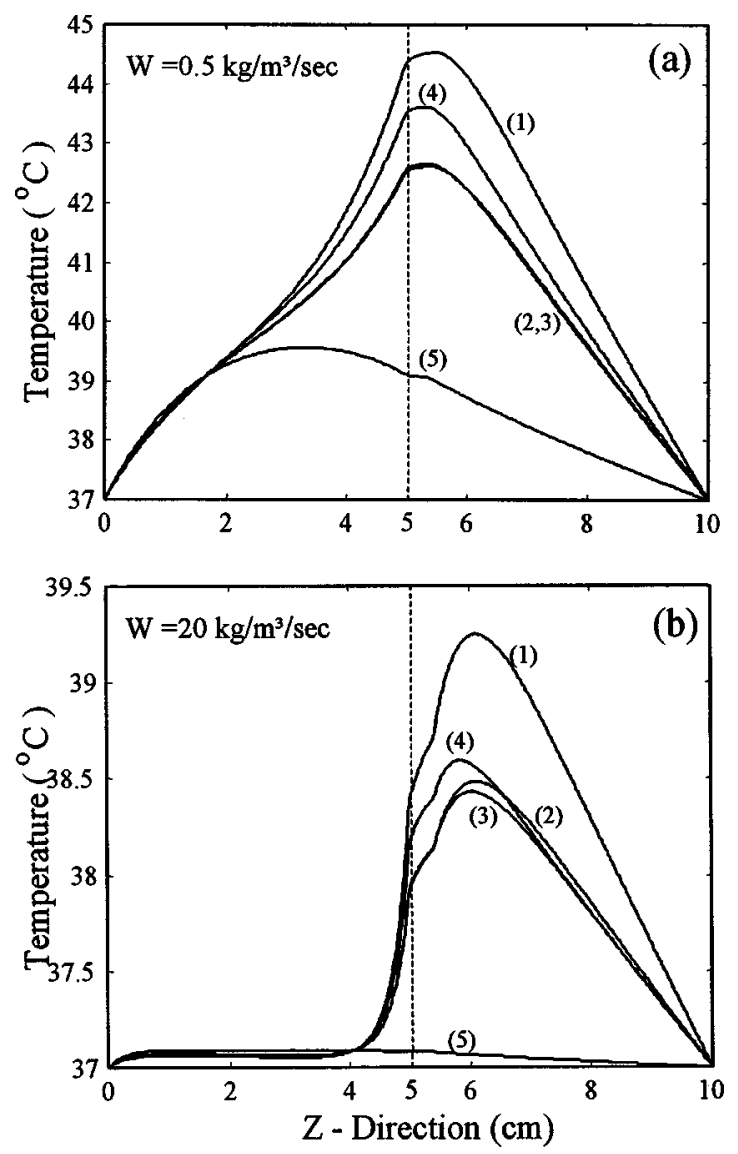

FIG. 4. Effect of blood perfusion in muscle on the temperature distribution. The blood perfusion for muscle varies from 0.5 to $20 \mathrm{~kg} \mathrm{~m}^{-3} \mathrm{~s}^{-1}$ with the other conditions maintained the same as Figs. 3(a) and 3(b).

increases. The ultrasound beam is totally reflected at $24.7^{\circ}$, and hence both the refracted longitudinal and shear waves drop to zero at that point. As the incident angle becomes greater than $24.7^{\circ}$, the refracted longitudinal wave disappears, and the magnitude of the reflected wave drops to its lowest value but the refracted shear wave jumps to its highest value. The second substantial change occurs when the incident angle approaches to $56.6^{\circ}$. As the incident angle is greater than $56.6^{\circ}$ the ultrasound beam totally reflects again. Figure 2(b) shows the energy partition ratios for the velocity of the longitudinal wave in bone equal to $2600 \mathrm{~m} / \mathrm{s}$, while the velocity of the shear wave is half of that value. Comparison with Figs. 2(a) and 2(b) reveals that the incident angle for total reflection increases and the magnitude of the reflected energy decreases when the ultrasound velocity in bone decreases.

To investigate the influence of the incident angle of the ultrasound beam at the interface of muscle and bone on the distributions of the SAR ratio and temperature in the tissues, several representative values taken from Fig. 2(a) and listed in Table II have been used. Figure 3(a) is the distribution of the SAR ratio for those cases in Table II using Eqs. (3) and (4) to calculate and scale the absorbed power deposition in the tissues for an ultrasound frequency of $1.0 \mathrm{MHz}$. The ultrasound attenuation at $1.0 \mathrm{MHz}$ is $5 \mathrm{~Np} \mathrm{~m}^{-1}$ for muscle, and 86.3 and $126.6 \mathrm{~Np} \mathrm{~m}^{-1}$ for the longitudinal and the shear waves in bone, respectively. ${ }^{38}$ The SAR ratio distribution shows that a sharp peak occurs at the interface of muscle and bone due to the much greater ultrasound absorption of the bone (refracted longitudinal and/or shear waves). The maximum of these peaks is the peak for the incident angle of $40^{\circ}$, at which $20 \%$ of the incident energy is reflected and the remainder is transmitted into the bone as shear wave. For case $1,100 \%$ of the incident energy is transmitted into the bone as longitudinal wave, so the ultrasound beam penetrates into the bone deeper than the other cases. For case 5, with a $60^{\circ}$ incident angle, there is no energy deposited in the bone, but the highest energy is in the muscle because the ultrasound beam is totally reflected at the interface. Figure 3(b) shows the corresponding temperature distribution for Fig. 3 (a). The input power is tuned for case 1 to reach a peak temperature of $41.5^{\circ} \mathrm{C}$, and then this input power level is fixed and used for all of the other cases. The peak temperature for case 5 (100\% reflection) occurs in the muscle, while the other peaks occur in the bone region beyond the interface of muscle and bone, instead of at the interface (the location of the peak of the SAR ratio). Figure 3(b) indicates that the peak temperature is higher for the case with a lower percentage of reflected energy, and hence case 1 with a $100 \%$ of transmitted energy produces the maximum of the peak temperatures. The temperature results obviously denote that the peak temperatures occur in the spongy/marrow region close to the cortical layer. The percentages of the reflected energy are equal for cases 2 and 3 . The difference between these two cases is that all transmitted ultrasound is in longitudinal waves for case 2 but for case 3 the shear wave is $27 \%$ of the total. However, there is no significant difference between the corresponding temperatures.

Ultrasound with 0.5 and $3.0 \mathrm{MHz}$ is used to investigate the effect of frequency on the temperature elevation across the muscle/bone interface. Figures 3(c)-3(d) show the temperature distributions for 0.5 and $3.0 \mathrm{MHz}$, respectively, with the other conditions maintained the same as Fig. 3(b). Comparison with Figs. 3(b) $-3($ d) indicates that a lower frequency ultrasound produces a higher temperature elevation deeper in the bone, but a lower temperature elevation in the muscle with the same input power level.

Blood perfusion always plays an important role in the temperature distribution during hyperthermia. Figures 4(a) and 4(b) are the temperature distributions with the conditions maintained the same as for Fig. 3(b) except the blood perfusion values for muscle are varied from 0.5 to $20 \mathrm{~kg} \mathrm{~m}^{-3} \mathrm{~s}^{-1}$. The input power level is fixed and used for all cases. The temperature distributions show that a muscle with lower perfusion results in higher temperatures not only in the muscle but also in the bone. Besides, the peak temperature shifts from the spongy/marrow region closer to the muscle/bone interface due to a weaker energy flow by conduction from the bone to the muscle. However, the ratio of temperature elevation for bone to muscle drops when the blood perfusion in muscle decreases.

The above study is based on the ultrasound attenuation 

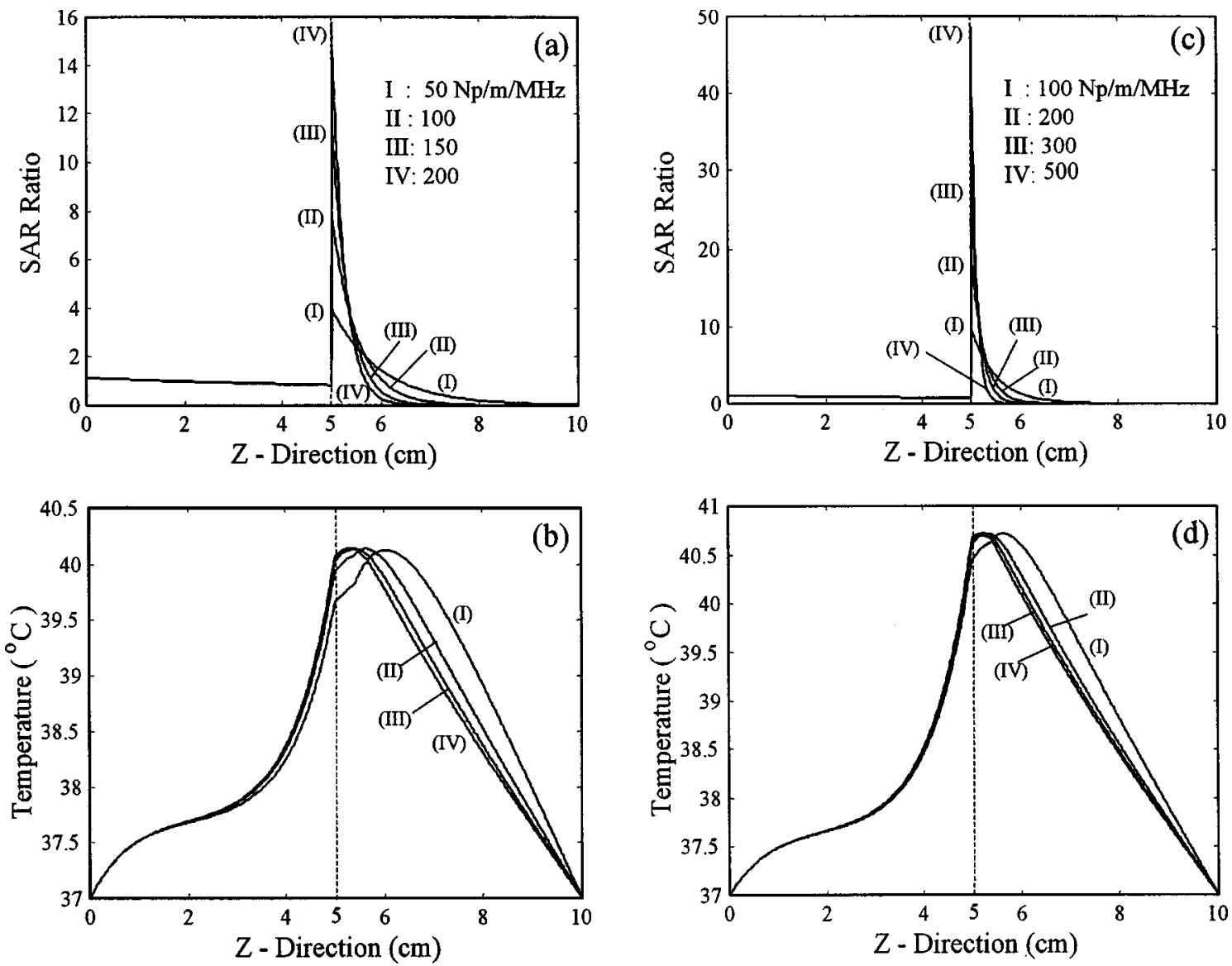

FIG. 5. Effect of acoustic absorption coefficient in bone on the distributions of the SAR ratio and temperature. (a) and (b) The energy partition of case 2 in Table II is used and the ultrasound attenuation for the refracted longitudinal wave $\left(\mu_{b}\right)$ of bone is equal to $50,100,150$, and $200 \mathrm{~Np} \mathrm{~m}^{-1} \mathrm{MHz}^{-1}$. (c) and (d) The energy partition of case 4 in Table II is used and the ultrasound attenuation for the refracted shear wave $\left(\mu_{b s}\right)$ in bone is equal to $100,200,300,500$, $\mathrm{Np} \mathrm{m}{ }^{-1} \mathrm{MHz}^{-1}$ with the other conditions maintained the same as Figs. 3(a) and 3(b).

coefficients of longitudinal and shear waves for bone ${ }^{38}$ of 86.3 and $126.6 \mathrm{~Np} \mathrm{~m}^{-1} \mathrm{MHz}^{-1}$, respectively, and $5 \mathrm{~Np} \mathrm{~m}^{-1}$ $\mathrm{MHz}^{-1}$ for muscle. In contrast, other investigators ${ }^{39-42}$ showed a large range of acoustic attenuation coefficients for bone tissues. The acoustic attenuation coefficient of bone is one of the crucial factors determining the distributions of the SAR ratio in tissues. It is necessary to study the influence of this factor on the distributions of the SAR ratio and temperature. Figures 5(a) and 5(b) present the results for case 2 in Table II, when the attenuation coefficient of the longitudinal wave for bone is varied from $50,100,150$, to $200 \mathrm{~Np} \mathrm{~m}^{-1}$ $\mathrm{MHz}^{-1}$ with the other conditions maintained the same as for Figs. 3(a) and 3(b). Figure 5(a) indicates that a greater attenuation coefficient of the longitudinal wave in bone results in a higher peak of the SAR ratio at the muscle/bone interface, whereas, for a lower attenuation coefficient, the ultrasound beam penetrates deeper into the bone. The corresponding temperature distribution as shown in Fig. 5(b) reveals that for a greater attenuation coefficient the peak temperature shifts closer to the muscle/bone interface. However, the values of all peak temperatures are about the same level due to the temperature elevation contributed by a higher absorbed power density, which is compensated for by the greater conduction. Figures 5(c) and 5(d) show the results, similar to
Figs. 5(a) and 5(b), for case 4 in Table II when the attenuation coefficient of shear wave for bone varies from 100, 200, 300 to $500 \mathrm{~Np} \mathrm{~m}^{-1} \mathrm{MHz}^{-1}$ with the other conditions maintained the same for Figs. 3(a) and 3(b). The SAR ratio peak at the muscle/bone interface is even higher and the SAR ratio distribution in the bone has even greater convergence to the cortical layer. The peak temperature is closer to the muscle/ bone interface for a higher attenuation coefficient of shear wave. Comparing Fig. 5(b) with Fig. 5(d), the temperature results indicate that a greater acoustic attenuation coefficient of bone results in a peak temperature closer to the muscle/ bone interface, with the temperature determined by the percentage of the ultrasound energy transmitted into the bone.

Figures 6(a) and 6(b) are the temperature distributions for case 2 in Table II when the input ultrasound power level for each set of perfusion and frequency is tuned to produce a peak temperature of $41.5^{\circ} \mathrm{C}$. Figure 6(a) reveals that a higher perfusion of muscle results in a lower temperature elevation in the muscle. This demonstrates that a highly perfused tumor in front of a bone is much more difficult to heat without overheating the bone. Conversely, a necrotic tumor could probably be heated without significant problems. The temperatures of Fig. 6(b) show that a higher frequency ultra- 

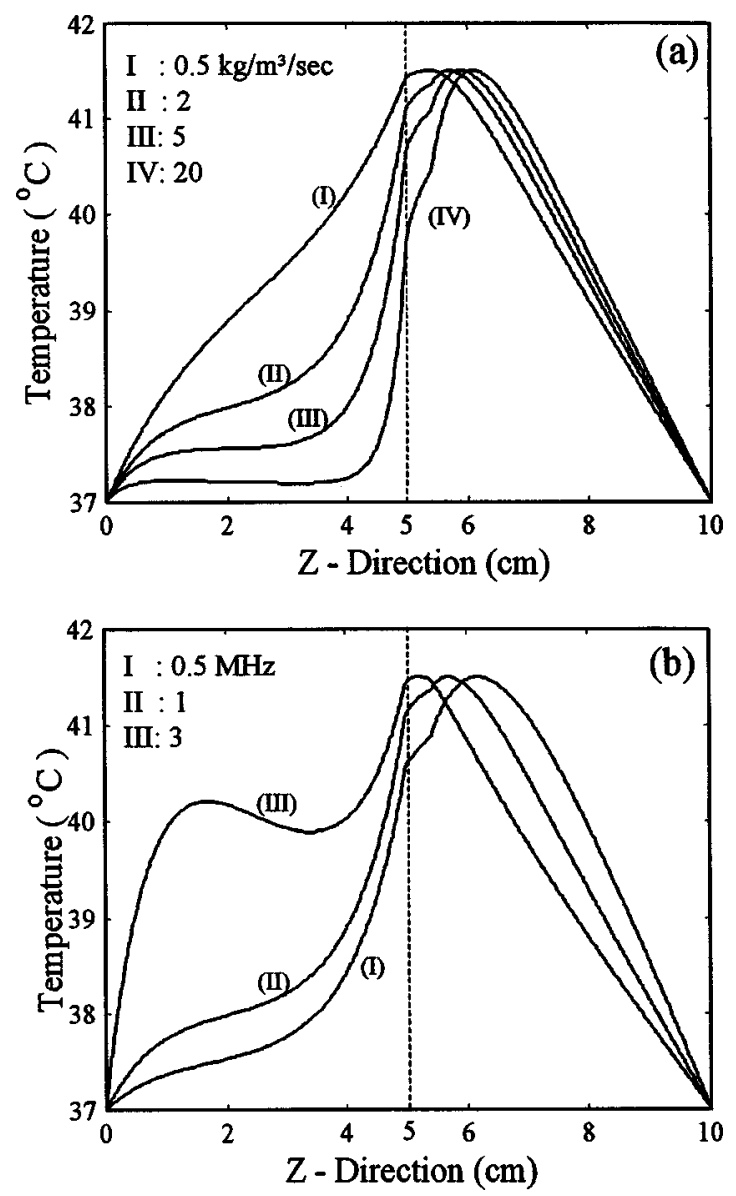

FIG. 6. Temperature distributions for the energy partition of case 2 in Table II. The input power level is tuned to produce a peak temperature of $41.5^{\circ} \mathrm{C}$. (a) Blood perfusion of muscle is from 0.5 to $20 \mathrm{~kg} \mathrm{~m}^{-3} \mathrm{~s}^{-1}$ for an ultrasound frequency of $1.0 \mathrm{MHz}$; (b) The ultrasound frequency is from 0.5 to 3 $\mathrm{MHz}$ for a muscle perfusion of $2 \mathrm{~kg} \mathrm{~m}^{-3} \mathrm{~s}^{-1}$.

sound can produce a greater temperature elevation in muscle. A tumor in front of a bone may be treatable without overheating the bone when an ultrasound beam is formed convergent to the tumor and then divergent, ${ }^{16,43}$ using an appropriate frequency and also considering the incident angle of the ultrasound beam to the bone surface (Figs. 2 and 3). The scanned focused ultrasound hyperthermia system ${ }^{17-21}$ or two-dimensional phased arrays of transducers ${ }^{22-24}$ can achieve this goal with a proper arrangement.

\section{DISCUSSION AND CONCLUSION}

The shape and incident angle of the ultrasound beam and the acoustic properties of the muscle and bone configure the pattern of absorbed power deposition. The conduction of the muscle and bone and the blood perfusion of muscle redistribute the absorbed power and produce a steady-state temperature distribution, which may be significantly different from the absorbed power deposition. The peak of temperature elevation appears at the interface or in the cortical layer very close to the interface of muscle and bone when the focal point of a focused ultrasound transducer is arranged close to the interface. ${ }^{1-3}$ This is due to the much higher absorption of the bone and the focused power in the focal zone. The study of the temperature elevation produced by this kind of power pattern is useful for ultrasound diagnostics, whereas the heating target for hyperthermia is a tumor, which may be located in front of a bone. The entire ultrasound beam is necessarily convergent to the tumor in order to deliver sufficient power to heat it and then divergent to avoid the overheating of the posttarget bone. ${ }^{43}$ Hence the power deposition for hyperthermia is greatly different from that produced by a single focused ultrasound transducer. The present work used a onedimensional model to theoretically calculate and examine the absorbed ultrasonic power deposition and the temperature elevation across the interface of muscle and bone. The proposed method can appropriately describe and analytically investigate the temperature elevation across the interface of muscle and bone for a large region heating, such as ultrasound hyperthermia or ultrasound physiotherapy, when the acoustic parameters and/or tissue properties are given.

This study of the SAR ratio and temperature distributions shows that the incident angle of the ultrasound beam at the muscle/bone interface, the acoustic attenuation of bone, and the blood perfusion of muscle are the crucial factors for absorbed power deposition and temperature elevation in tissues. Previous investigators $3,8,9,14$ have suggested that a specific or large incident angle $\left(24.7^{\circ}\right.$ or $>56.6^{\circ}$ in this study) of the ultrasound beam can be used to minimize the ultrasound energy transmitted into the bone, thus effectively preventing the overheating of the bone. When these incident angles cannot be arranged for the ultrasound beam during hyperthermia, a small angle that can result in a relatively higher reflection is suggested for use (Fig. 2). A higher reflection of the ultrasound beam means that a smaller amount of ultrasound energy penetrates into the bone and a lower temperature elevation occurs in the bone (Fig. 3). In addition to the incident angle of ultrasound beam, the ultrasound frequency is another control factor that can be used to effectively reduce the peak of the SAR ratio at the muscle/bone interface and/or to shorten the penetration depth of the ultrasound beam in the bone (Fig. 3). As a result, the temperature elevation in the bone drops dramatically for a higher frequency when the ultrasound beam propagates from the muscle side with the same input power level. This indicates that an appropriate frequency and incident angle of the ultrasound beam should be selected in order to deliver sufficient energy to heat a tumor and not to overheat the bone behind the tumor. ${ }^{9,14,15,43}$ The incident angle of the ultrasound beam considered in this study is directly located at the interface of muscle and bone, whereas it is at the interface of fat and muscle for the three layer (fat/muscle/bone) model proposed by Chan et al. ${ }^{8,9}$ These two incident angles can be transferred from one to the other using Snell's law.

The distributions of the SAR ratio indicate that the peak of absorbed power density is always at the interface of muscle and bone except for the case of total reflection. Immediately after the exposure of ultrasound power begins, the temperature rise is linearly proportional to the local SAR and the function of temperature rise resembles the distribution of 
the SAR ratio. Within several seconds, thermal conduction becomes very significant and broadens the thermal distribution across the interface of muscle/bone. The location of the peak temperature is not only determined by the SAR ratio, which is related to the ultrasound frequency and the incident angle of ultrasound beam (Fig. 3), but also by the blood perfusion of muscle (Fig. 4). The energy taken away by blood perfusion is related to the magnitude of perfusion as well as the temperature difference of local tissue and arterial blood. Under the same conditions, a higher muscle perfusion results in a lower temperature elevation in the muscle, thus causing a greater temperature drop at the muscle/bone interface due to the stronger thermal conduction from bone to muscle. Hence the peak temperature drops and shifts from the interface/cortical layer to the spongy/marrow region for a higher perfused muscle (Fig. 4). In addition, the ultrasound attenuation of bone is the other tissue property that affects the location of the peak temperature. The peak temperature shifts closer to the muscle/bone interface for a bone with a higher ultrasound attenuation (Fig. 5).

The ultrasound wave was assumed to smoothly transmit through the second interface (cortical layer/spongy bone) in the above study and Eq. (3b), which neglects the effect of the second interface, was used to describe the absorbed power deposition in the bone region $\left(z_{b} \leqslant z \leqslant z_{e}\right)$. When the second interface is considered as a sharp change in acoustic impedance and normal incidence $(\alpha=0)$ for the ultrasound beam, the absorbed power density in the bone region is

$$
q(z)=\left\{\begin{array}{cc}
2 \mu_{b}(1-R) I(0) \cdot e^{-2 \mu_{m} z_{b}} \cdot\left[e^{-2 \mu_{b}\left(z-z_{b}\right)}+R^{\prime} \cdot e^{-2 \mu_{b}\left[2 z_{c}-\left(z+z_{b}\right)\right]}\right] & \text { for } z_{b} \leqslant z \leqslant z_{c} \\
2 \mu_{b}(1-R) I(0) \cdot e^{-2 \mu_{m} z_{b}} \cdot e^{-2 \mu_{b}\left(z_{c}-z_{b}\right)} \cdot\left[\left(1-R^{\prime}\right) e^{-2 \mu_{b}\left(z-z_{c}\right)}\right] & \text { for } z_{c} \leqslant z \leqslant z_{e}
\end{array}\right.
$$

where $R^{\prime}$ is the energy ratio of the reflected wave to the incident wave at the second interface and can be obtained by Eq. (2a). Based on the density differences of the muscle/ cortical layer/spongy region and the velocity difference of the muscle/cortical layer, $R^{\prime}$ is about equal to 0.12 for a 2300 $\mathrm{m} / \mathrm{s}$ acoustic velocity in the spongy bone. The resulting SAR ratio in the cortical layer is $3.6 \%$ higher at $z_{b}^{+}$(the interface of muscle/cortical layer) and $12 \%$ higher at $z_{c}^{-}$(the interface of cortical layer/spongy bone) than those without considering the second reflection, while the SAR ratio in the spongy/ marrow region decreases $12 \%$. Hence the location of maximum temperature elevation may shift from the spongy/ marrow region to the cortical layer for a sharp change of acoustic impedance at the second interface.

\section{ACKNOWLEDGMENTS}

The authors would like to thank the National Science Council of the Republic of China for partially supporting this research under Contract No. NSC 89-2213-E-002-042.

\footnotetext{
a) Author to whom all coresspondence should be addressed: Ming-Jium Shieh, M.D., Ph.D., No. 1, Sec. 1, Jen-Ai Road, Taipei, Taiwan; Fax: (886) 2 23940049; Electronic mail: kcju@ipmc.ee.ntu.edu.tw

${ }^{1}$ J. Wu and G. Du, "Temperature elevation generated by a focused Gaussian ultrasonic beam at a tissue-bone interface," J. Acoust. Soc. Am. 87, 2748-2755 (1990).

${ }^{2}$ NCRP Report No. 113, Exposure Criteria for Medical Diagnostic Ultrasound: I. Criteria based on the Thermal Mechanisms (NCRP Publication, Bethesda, MD, 1992), pp. 52-60.

${ }^{3}$ M. Fujii, K. Sakamoto, Y. Toda, A. Negishi, and H. Kanai, "Study of the cause of the temperature rise at the muscle-bone interface during ultrasound hyperthermia,"' IEEE Trans. Biomed. Eng. 46, 494-504 (1999).

${ }^{4}$ P. A. Nelson, J. F. Herrick, and F. H. Krusen, "Temperature produced in bone marrow, bone and adjacent tissues by ultrasound diathermy," Arch. Phys. Med. 31, 687-695 (1950).

${ }^{5}$ J. F. Lehmann, B. J. deLateur, and D. R. Silverman, "Selective heating effects of ultrasound in human being," Arch. Phys. Med. Rehabil. 47, 331-339 (1966).
}

${ }^{6}$ J. F. Lehmann, B. J. deLateur, C. G. Warren, and J. S. Stonebridge, "Heating produced by ultrasound in bone and soft tissue," Arch. Phys. Med. Rehabil. 48, 397-401 (1967).

${ }^{7}$ J. F. Lehmann, J. S. Stonebridge, B. J. deLateur, C. G. Warren, and E. Halar, "Temperatures in human thighs after hot pack treatment followed by ultrasound,"' Arch. Phys. Med. Rehabil. 59, 472-475 (1978).

${ }^{8}$ A. K. Chan, R. A. Sigelmann, A. W. Guy, and J. F. Lehmann, "Calculation by the method of finite differences of the temperature distribution in layered tissues," IEEE Trans. Biomed. Eng. 20, 86-90 (1973).

${ }^{9}$ A. K. Chan, R. A. Sigelmann, and A. W. Guy, "Calculations of therapeutic heat generated by ultrasound in fat-muscle-bone layers," IEEE Trans. Biomed. Eng. 21, 280-284 (1974).

${ }^{10}$ E. G. Moros, X. Fan, and W. L. Straube, "Ultrasound power deposition model for the chest wall," Ultrasound Med. Biol. 25, 1275-1287 (1999).

${ }^{11}$ J. B. Marmor, D. Pounds, T. B. Postic, and G. M. Hahn, "Treatment of superficial human neoplasms by local hyperthermia induced by ultrasound," Cancer 43, 188-197 (1979).

${ }^{12}$ P. M. Corry, B. Barlogie, E. J. Tilchen, and E. P. Armour, "Ultrasound induced hyperthermia for the treatment of human superficial tumors," Int. J. Radiat. Oncol., Biol., Phys. 8, 1225-1229 (1982).

${ }^{13}$ P. Fessenden, E. R. Lee, T. L. Anderson, J. W. Strohbehn, J. L. Meyer, T. V. Samulski, and J. R. Marmor, "Experience with a multi-transducer ultrasound system for localized hyperthermia of deep tissues," IEEE Trans. Biomed. Eng. 31, 126-135 (1984).

${ }^{14} \mathrm{~K}$. Hynynen and D. DeYoung, "Temperature elevation at muscle-bone interface during scanned, focused ultrasound hyperthermia," Int. J. Hyperthermia 4, 267-279 (1988).

${ }^{15}$ S. J. Tu, K. Hynynen, and R. B. Roemer, "Simulation of bi-directional ultrasound hyperthermia treatments of neck tumors,' Int. J. Hyperthermia 10, 707-722 (1994).

${ }^{16}$ W.-L. Lin, J.-Y. Yen, Y.-Y. Chen, K.-W. Jin, and M.-J. Shieh, "Relationship between acoustic aperture size and tumor conditions for external ultrasound hyperthermia," Med. Phys. 26, 818 (1999).

${ }^{17}$ G. H. Nussbaum, W. L. Straube, M. D. Drag, G. L. Melson, B. Emami, V. Sathiaseelan, E. Seppi, and E. Shapiro, "Potential for localized, adjustable deep heating in soft-tissue environments with a 30-beam ultrasonic hyperthermia system,'” Int. J. Hyperthermia 7, 279-299 (1991).

${ }^{18}$ W.-L. Lin, R. B. Roemer, E. G. Moros, and K. Hynynen, "'Optimization of temperature distributions in scanned, focused ultrasound hyperthermia,' Int. J. Hyperthermia 8, 61-78 (1992).

${ }^{19}$ K. Lindsley, P. R. Stauffer, P. Sneed, R. Chin, T. L. Phillips, E. Seppi, E. Shapiro, and S. Henderson, "Heating patterns of the Helios ultrasound hyperthermia system,' Int. J. Hyperthermia 9, 675-684 (1993).

${ }^{20}$ A. W. Dutton, R. B. Roemer, and F. A. Gibbs, "A clinically integrated scanned focused ultrasound hyperthermia," in Proceedings of the 7th 
International Congress on Hyperthermic Oncology (Rome, Italy, 1996), Vol. 1, p. 92.

${ }^{21}$ W.-L. Lin, Y.-Y. Chen, S.-Y. Lin, J.-Y. Yen, M.-J. Shieh, and T.-S. Kuo, "Optimal configuration of multiple-focused ultrasound transducers for external hyperthermia," Med. Phys. 26, 2007-2016 (1999).

${ }^{22}$ C. A. Cain and S. Umemura, "Concentric-ring and sector vortex phased array applicators for ultrasound hyperthermia therapy," IEEE Trans. Microwave Theory Tech. 34, 542-551 (1986).

${ }^{23}$ E. S. Ebbini and C. A. Cain, "A spherical-section ultrasound phased array applicator for deep localized hyperthermia," IEEE Trans. Biomed. Eng. 38, 634-643 (1991).

${ }^{24}$ R. J. McGough, E. S. Ebbini, and C. A. Cain, "Treatment planning for hyperthermia with ultrasound phased arrays," IEEE Trans. Ultrason. Ferroelectr. Freq. Control 43, 1074-1084 (1996).

${ }^{25}$ E. G. Moros, R. B. Roemer, and K. Hynynen, "Pre-focal plane hightemperature regions induced by scanning focused ultrasound beams," Int. J. Hyperthermia 6, 351-366 (1990).

${ }^{26}$ B. A. Haken, L. A. Frizzell, and E. L. Carstensen, "Effect of mode conversion on ultrasonic heating at tissue interfaces," J. Ultrasound Med. 11, 393-405 (1992).

${ }^{27}$ W. G. Mayer, "Energy deposition of ultrasonic waves at flat boundaries," Ultrasonics 3, 62-68 (1965).

${ }^{28} \mathrm{~K}$. Hynynen, "Biophysics and technology of ultrasound hyperthermia," in Methods of External Hyperthermic Heating, edited by M. Gautherie (Springer, Berlin, 1990), pp. 61-116.

${ }^{29} \mathrm{H}$. H. Pennes, "Analysis of tissue and arterial blood temperatures in the resting human forearm," J. Appl. Phys. 1, 93-122 (1948).

${ }^{30} \mathrm{H}$. Arkin, X. Xu, and K. R. Holmes, "Recent developments in modeling heat transfer in blood perfusion tissues," IEEE Trans. Biomed. Eng. 41, 97-107 (1994).

${ }^{31}$ R. B. Roemer, "Thermal dosimetry," in Thermal Dosimetry Planning, edited by M. Gautherie (Springer, Berlin, 1990), pp. 119-214.

${ }^{32}$ J. C. Chato, "Fundamentals of bioheat transfer," in Thermal Dosimetry and Treatment Planning, edited by M. Gautherie (Springer, Berlin, 1990), pp. 1-56.
${ }^{33}$ H. F. Bowman, "Heat transfer mechanism and thermal dosimetry," Natl. Cancer Inst. Monograph 61, 437-445 (1982).

${ }^{34}$ J. C. Chato, "Selected thermophysical properties of biological materials," in Heat Transfer in Medicine and Biology, Analysis and Applications, edited by A. Shitzer and R. C. Eberhart (Plenum, New York, 1985), pp. 413-418.

${ }^{35}$ R. G. Gordon, R. B. Roemer, and S. M. Horvath, “A mathematical model of the human temperature regulatory system - transient cold exposure response,"' IEEE Trans. Biomed. Eng. 23, 434-444 (1976).

${ }^{36}$ K. M. Sekins, and A. F. Emery, "Thermal science for physical medicine," in Therapeutic Heat and Cold, edited by J. F. Lehmann (Williams and Wilkins, Baltimore, 1982), pp. 70-132.

${ }^{37}$ R. K. Jain, "Bio-heat transfer: Mathematical models of thermal systems," in Hyperthermia Cancer Therapy, edited by F. C. Storm (Hall, Boston, 1983), pp. 9-46.

${ }^{38} \mathrm{~J}$. Wu and F. Cubberley, "Measurement of velocity and attenuation of shear waves in bovine compact bone using ultrasonic spectroscopy," Ultrasound Med. Biol. 23, 129-134 (1997).

${ }^{39}$ S. A. Goss, R. L. Johnson, and F. Dunn, "Comprehensive compilation of empirical ultrasonic properties of mammalian tissues," J. Acoust. Soc. Am. 64, 423-457 (1978).

${ }^{40}$ S. A. Goss, L. A. Frizzell, and F. Dunn, "Ultrasonic absorption and attenuation of high frequency sound in mammalian tissues," Ultrasound Med. Biol. 5, 181-186 (1979).

${ }^{41}$ S. A. Goss, R. L. Johnson, and F. Dunn, "Compilation of empirical ultrasonic properties of mammalian tissues, II,' J. Acoust. Soc. Am. 68, 93-108 (1980).

${ }^{42}$ L. A. Frizzell and E. Carstensen, "Shear properties of mammalian tissues at low megahertz frequencies," J. Acoust. Soc. Am. 60, 1409-1411 (1976).

${ }^{43}$ W.-L. Lin, C.-T. Liauh, J.-Y. Yen, Y.-Y. Chen, and M.-J. Shieh, "Treatable domain and optimal frequency for brain tumors during ultrasound hyperthermia," Int. J. Radiat. Oncol., Biol., Phys. 46, 239-247 (2000). 